\title{
Skillfully Using Rural Domestic Resources to Conduct Regional Activities: A Case Study of Eastern Hubei Province
}

\author{
Lu Xue \\ Huanggang Normal University, Hubei, China, 438000
}

Keywords: indigenous resources; regional activities; young children

Abstract: Regional activities are games with a learning nature that children play under the premise of their own will. The characteristic of land resources in this area is the cultivation of edible fungi. Therefore, on this basis, this article discusses how to use local resources to carry out the practical exploration of characteristic regional activities.

\section{Introduction}

East Hubei is the eastern part of Hubei Province, Hubei Province (short for Hubei Province). The Eastern and Southern Dynasties refer mainly to the Huanggang region, which refers broadly to the three eastern cities of Hubei Province, including Huanggang City, Huangshi City, and Ezhou City. The division of the administrative districts in eastern Hubei is now divided into three prefecture-level cities: Huanggang, Ezhou and Huangshi. The total area of 23558 square kilometers. The total population of 10989640 people. The practice of using regional local resources to carry out regional activities in eastern Hubei has important significance and influence.

\section{Precautions for using local resources to carry out special regional activities}

\subsection{Safety}

The "Kindergarten Education Guidance Outline" stipulates: "Kindergartens must place the protection of children's lives and promote the health of children's health at the top of their work." Therefore, we must pay attention to safety in the use of local resources to carry out special regional activities. Because young children are in a state of being ignorant of the world, sometimes it is not clear what they can eat, what cannot eat, and there is no awareness of self-protection. For example, because of curiosity about indigenous resources, mushrooms, they often bite mushrooms in the game. This is very prone to accidents. Therefore, in addition to giving students a sense of inability to eat mushrooms, they should also choose non-toxic and tasteless. Mushroom species that are not dangerous to early childhood health and are strictly cleaned and disinfected to ensure the safety of young children.

Avoid food poisoning due to ingestion. From the data, it can be found that high school students who know more about local culture in eastern Hubei account for $49 \%$ of the total, and students who do not understand and students who generally understand account for $51 \%$. It shows that the 
students surveyed have a poor understanding of local culture in Chengdu.

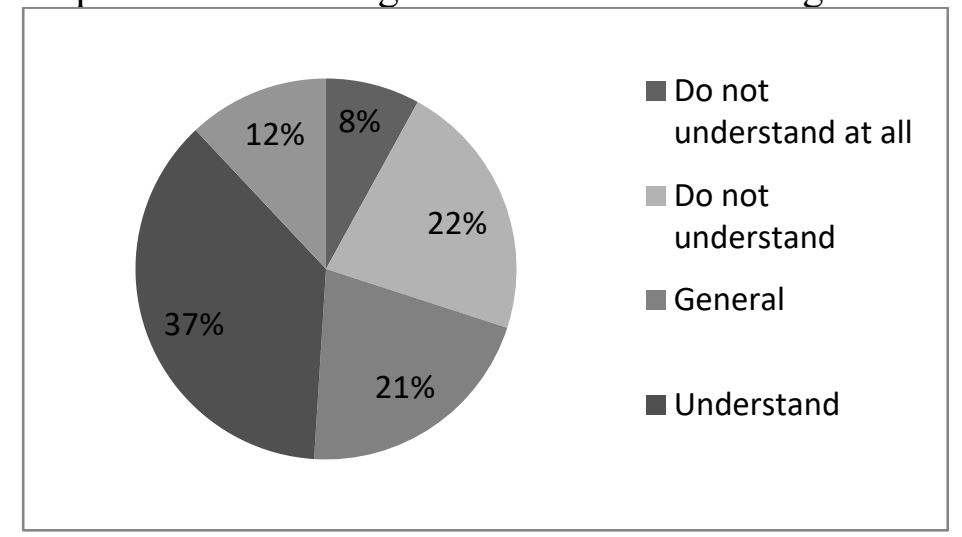

Fig.1 Students' understanding of local culture in Eastern Hubei

\subsection{Hierarchical}

The use of local resources should pay attention to levels, mainly in the horizontal and vertical aspects. Although young children are similar in age, but because each individual has its own development characteristics, so the requirements for land resources and materials are not the same, so you can plant different species in the same area, easy for children to choose according to their own interests and hobbies Different materials to learn and explore. For example, local edible fungus cultivation can choose different types. Mushrooms, straw mushrooms, mushrooms, fungus, and white fungus can all be used. Since young children are in a period of rapid development, the input of materials can not be unplanned, and it should be divided into batches and targeted in order to meet the development characteristics of children. For example, in the area of edible fungus materials, you can first put the more common mushrooms and fungus in your life, and then put in the less common mushrooms, straw mushrooms, and white fungus.

\subsection{Inquiry}

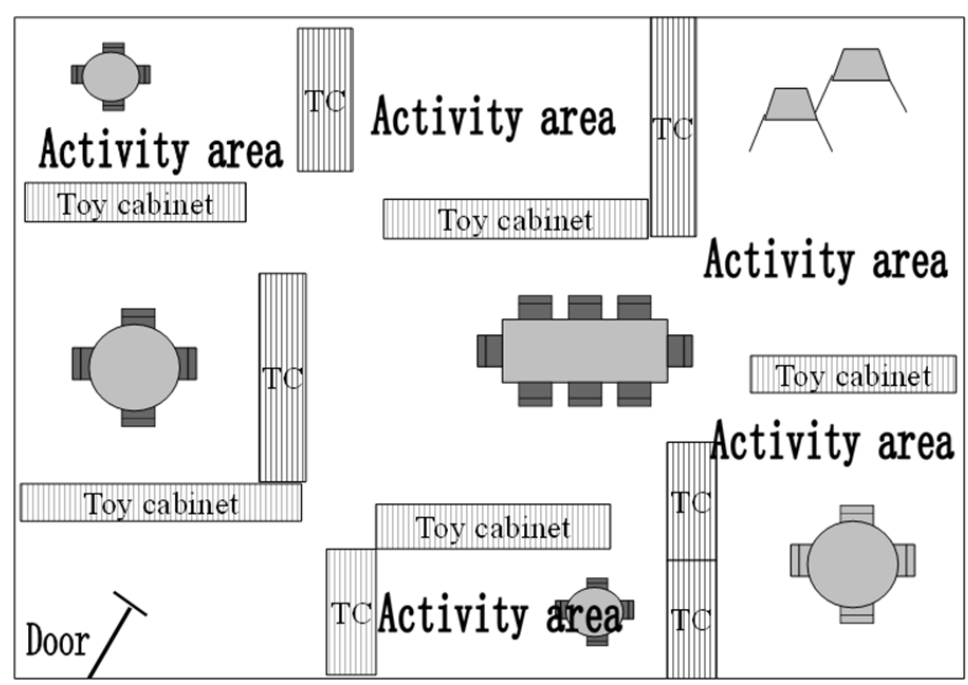

Fig. 2 Planning of Kindergarten Activities Based on Eastern Hubei Resources

The exploration of land resource materials can enhance children's observation ability, thinking ability, hands-on ability and practical ability. Inquiry is not simply a matter of operation or practice. 
It is a hands-on exercise based on mental thinking. It is the interaction between young children and land resources materials and the environment. For example, giving young children several varieties of edible mushrooms allows them to identify the appearance, name and way of eating. This is not a typical inquiry activity. Give the children several different types of edible fungus seeds, guide the students to plant and observe the growth process, record the growth state, organize the young children to pick and produce foods after the maturity, and also display the picked fruits so that they can be observed and discussed at any time This is the true form of discussion. Therefore, the provision of land resource materials should focus on the exploration of materials, so as to stimulate children's interest and make activities and games more long-term, which helps to enhance children's observation ability, thinking ability, hands-on ability and practical ability.

\section{The practice of using local resources to develop characteristic regional activities}

\subsection{Clever use of local resources to cultivate children's creativity}

In regional activities, the operation of land resource materials can better stimulate children's creativity, and in the process experience the tremendous value of simple things. So that children really like to explore, learn to explore and master exploration. Firstly, the design of regional activities is conducive to young children's initiative and is the basic condition for children's creativity. Therefore, at this stage, we must first guide children to become interested in and familiar with the materials. For example, we can use fungus to develop characteristic regional activities. We can first give students pictures of some edible fungi and foods for edible fungus, helping students to enhance the information of regional activities. desire. By using the engineering PRB calculation concept for resource utilization, it can be used for reference to the development and utilization of rural resources:

$$
\begin{aligned}
& \text { CQI_Per_Frame } \\
& P R B \\
& S R S_{-} \text {Per_Frame } \\
& P R B
\end{aligned}
$$

In the above formula, CQI and SRS represent different local resources, which have an important role in the promotion and practice of regional activities. At the same time, teachers can also cherish materials with corresponding infiltration culture, and do not waste material awareness. Second, teachers and young children can work together to plan the layout of regional activities. In this process, children's creativity is fully reflected, and the independent creation of the environment can also make young children have the feeling of being a master. At the same time, the relationship between teachers and students will further warm up, which is conducive to the next regional activities. For example, how can the mushroom cultivation land be divided into pieces, how the greenhouses are constructed, and the type of description of wooden signs can be given to students. Finally, we must guide young children in the process of regional activities and seize the opportunity to actively develop young children's imagination and creativity. When assessing young children, teachers should proceed from the following aspects: Conventional habits: habits of entering the district, habits of picking and placing materials, habits of tidying up, and so on. Improve experience: Including children's experience in areas such as cognition, ability, and emotion.

\subsection{Actively organize the development of integrated and regional practical activities}

To carry out practical activities, we must first clarify the objectives of the activities. That is, there must be a direction between activities. We must not be confused and have no goals. The quality of activities will be greatly reduced, and young children will not be able to obtain any important life in 
the activities. Feelings. Activities can be combined with specific situations to propose different goals. For example, when edible fungi are used for regional activities, because the fungi they cultivate are edible, the goal of teaching can be defined as the identification and consumption of edible fungi in life. Second, it is necessary to enrich the forms of activities.

There are many kinds of edible fungi. Although young children may have seen these fungi in their lives, they do not know their names, and they are even less aware of their living habits and food value. Teachers can organize activities such as the cultivation of small edible fungi by edible mushrooms. Help young children develop their hands-on abilities and observation skills by personally cultivating edible fungus, so as to better understand some knowledge of edible fungi and increase their life experience. Finally, we must arrange the display of results. This is because different edible fungi have different characteristics. For example, fungus is black, ordinary mushrooms are brown, and if the mushroom is round, the mushroom is umbrella-shaped.

\section{Summary}

Regional activities are an important part of the new kindergarten curriculum and are highly valued by experts and teachers. The purpose of the regional activities is to better promote the children's physical and mental development in a comprehensive and harmonious manner, and must not be in a form.

\section{Acknowledgement}

1) Research on the utilization of local resources in regional activities of rural kindergartens -- a case study of eastern hubei province (Hubei education department humanities and social science research youth project. Project number: 03201702102)

2) Research on the utilization of characteristic curriculum resources in the regional activities of kindergartens in Eastern Hubei (Eastern hubei education and cultural research center research projects. Project number: 201607103)

\section{References}

[1] Momba M, Tyafa Z, Makala N, et al. Safe drinking water still a dream in rural areas of South Africa. Case Study: The Eastern Cape Province[J]. Water S A, 2006, 32(5):200-201.

[2] Gemelli A, Mancini A, Longhi S. GIS-based energy-economic model of low temperature geothermal resources: A case study in the Italian Marche region[J]. Renewable Energy, 2011, 36(9):2474-2483.

[3] Zhu Q, Lowe E A, Wei Y A, et al. Industrial Symbiosis in China: A Case Study of the Guitang Group[J]. Journal of Industrial Ecology, 2007, 11(1):31-42. 